\title{
Características nociceptivas e histomorfométricas de nervos medianos de ratos com obesidade induzida pelo glutamato monossódico
}

\author{
Nociceptive and histomorphometric characteristics of median nerves of rats \\ with obesity induced by monosodium glutamate
}

\author{
Josinéia Gresele Coradini' ${ }^{1}$, Jhenifer Karvat ${ }^{2}$, Rose Meire Costa Brancalhão ${ }^{3}$, Lucinéia de Fátima Chasko Ribeiro ${ }^{4}$, \\ Maria Lúcia Bonfleur ${ }^{5}$, Gladson Ricardo Flor Bertolini ${ }^{6}$ \\ ${ }^{1}$ Mestre em Biociências e Saúde pela Universidade Estadual do Oeste do Paraná (Unioeste). \\ ${ }^{2}$ Mestranda em Biociências e Saúde pela Unioeste. Cascavel, PR. \\ 3 Doutora em Zoologia pela Universidade Federal do Paraná. Professora do mestrado em Biociências e Saúde da Unioeste. Cascavel, PR. \\ ${ }^{4}$ Doutora em Ciências Biológicas (Biologia Celular) pela Universidade Estadual de Maringá. Professora do Mestrado em Biociências e Saúde da Unioeste. Cascavel, PR. \\ ${ }^{5}$ Doutora em Biologia Funcional e Molecular pela Universidade Estadual de Campinas. Professora do mestrado em Biociências e Saúde da Unioeste. Cascavel, PR. \\ ${ }^{6}$ Doutor em Ciências da Saúde Aplicadas ao Aparelho Locomotor pela Faculdade de Medicina de Ribeirão Preto da Universidade de São Paulo. Professor do Mestrado \\ em Biociências e Saúde da Unioeste. Cascavel, PR.
}

\section{RESUMO}

Objetivos: Comparar a nocicepção e, histomorfometricamente, a secção transversa de nervos periféricos de ratos Wistar submetidos ao modelo de obesidade induzida pelo glutamato monossódico com animais controle.

Métodos: Foram utilizados ratos Wistar, divididos em grupo obeso e grupo controle. Desde o nascimento, durante os primeiros cinco dias de vida, os ratos do grupo obeso receberam uma injeção diária subcutânea de glutamato monossódico (4g/kg de peso corporal/dia), enquanto os ratos do grupo controle receberam solução salina hiperosmótica $(1,25 \mathrm{~g} / \mathrm{kg}$ de peso corporal $/$ dia $)$. Foi avaliada a nocicepção, pelo limiar de retirada do membro, com uso de analgesímetro digital tipo Von Frey, com o estímulo dado na região palmar da pata anterior direita. A primeira avaliação foi realizada cerca de 20 dias antes da eutanásia, sendo a segunda avaliação realizada no dia anterior ao da eutanásia. Posteriormente, foi dissecado o nervo mediano, na região do cotovelo e processado com cortes transversais para análise histológica. As variáveis analisadas foram: número de axônios por campo; diâmetros dos axônios, das fibras e da bainha de mielina, e coeficiente G. Os resultados foram analisados por meio do teste $t$ de Student para amostras independentes e t pareado, com nível de significância de 5\%.

Resultados: Foram estudados 14 ratos, sendo sete no grupo obeso e sete no grupo controle. A avaliação da nocicepção mostrou que os animais do grupo obeso apresentavam menor limiar de retirada. Para os dados histomorfométricos, os resultados não mostraram diferenças significativas entre os dois grupos estudados.

Conclusões: Os animais obesos apresentaram menor limiar nociceptivo, porém não houve diferenças morfométricas dos nervos medianos entre animais submetidos ao modelo de obesidade induzida pelo glutamato monossódico e os controles.

DESCRITORES: OBESIDADE; MEDIÇÃO DA DOR; NERVO MEDIANO; RATOS WISTAR.

\section{ABSTRACT}

Aims: To compare nociception and, histomorphometrically, the transverse section of peripheral nerves (median) of Wistar rats submitted to obesity model induced by monosodium glutamate with control animals.

Methods: Fourteen Wistar rats divided into control and obese groups were used. During the five first days since birth the rats from obese group received a daily subcutaneous injection of monosodium glutamate $(4 \mathrm{~g} / \mathrm{kg}$ body weight/day), while the control group received hypertonic saline $(1.25 \mathrm{~g} / \mathrm{kg}$ body weight/day). Nociception was evaluated by the withdrawal threshold of the limb, using digital analgesymeter type Von Frey, with the stimulus given in the palmar region of the right hind paw. The first assessment was carried out about 20 days before euthanasia, and the second assessment was performed on the day before euthanasia. Subsequently the median nerve was dissected in the elbow region and processed with cross sections for histological analysis. The analyzed variables were: number of axons per field; axons, fibers and myelin sheath diameters, and $\mathrm{G}$ coefficient. The results were analyzed using the $t$ test for independent samples and paired $t$ test, with a significance level of $5 \%$. Results: Fourteen rats were assessed, being seven of the obese group and seven of control group. The evaluation of nociception showed that the animals of the obese group had lower withdrawal threshold. For histomorphometric data, the results showed no significant differences between the two groups.

Conclusions: The obese animals showed lower nociceptive threshold, however, there were no morphometric differences of the median nerves between animals subjected to the model of obesity induced by monosodium glutamate and the control group.

KEY WORDS: OBESITY; PAIN MEASUREMENT; MEDIAN NERVE; RATS, WISTAR. 


\section{INTRODUÇÃO}

A obesidade pode ser caracterizada como o acúmulo de gordura corporal, generalizado ou localizado, que acarreta prejuízos à saúde de um indivíduo. ${ }^{1}$ Além dos fatores socioculturais, as alterações no balanço energético que causam a obesidade podem estar relacionadas a fatores genéticos, metabólicos, endócrinos e neurais. ${ }^{2}$ Alterações no hipotálamo, região que exerce um papel chave na homeostase energética e no controle do peso corporal, levam ao desenvolvimento de distúrbios metabólicos, ganho de peso e obesidade conhecida como obesidade hipotalâmica. ${ }^{3}$ A obesidade está associada a alguns distúrbios metabólicos que incluem intolerância à glicose, hipertensão arterial, dislipidemia e diabetes mellitus tipo $2 .{ }^{4}$

A neuropatia periférica diabética é uma das mais frequentes complicações da diabetes mellitus, sendo considerada a principal causa de morbidade e mortalidade entre diabéticos; está frequentemente associada com dor debilitante e lidera as amputações não traumáticas. ${ }^{5-7}$ Existem evidências epidemiológicas e laboratoriais para sustentar outros fatores, além da hiperglicemia, para o desenvolvimento da neuropatia periférica diabética, incluindo obesidade, hipertensão, dislipidemia, inflamação e resistência à insulina. ${ }^{5,8} \mathrm{~A}$ neuropatia periférica pode ocorrer tanto em pacientes pré-diabéticos quanto em diabéticos, e também pode ser desenvolvida em modelos animais. ${ }^{9}$ Tal neuropatia é associada com perda sensitiva e degeneração de todos os tipos de fibras em nervos periféricos, sendo observada, em animais de experimentação, uma fase de hiperalgesia seguida por hipoestesia. ${ }^{10}$

Para melhor compreensão dos mecanismos envolvidos com a obesidade e suas comorbidades, são utilizados diferentes modelos animais experimentais obesos, diabéticos e pré-diabéticos. Um modelo amplamente utilizado é o de obesidade induzida por lesão hipotalâmica através de injeções de glutamato monossódico (MSG). ${ }^{11}$ Quando o MSG é administrado no período neonatal, em roedores, ocorrem lesões no núcleo arqueado do hipotálamo, na eminência mediana, levando à obesidade nesses animais, na vida adulta. ${ }^{11}$ A obesidade hipotalâmica MSG em roedores simula a obesidade hipotalâmica em humanos e também está associada à hiperinsulinemia, com posterior resistência à insulina. ${ }^{12-14}$ Entretanto, esses animais são considerados pré-diabéticos, visto que são normoglicêmicos frente à hiperinsulinemia. ${ }^{15}$

A neuropatia periférica é um grande problema para a população diabética, sendo a obesidade um dos fatores de risco para essa condição. No modelo experimental de obesidade por MSG ocorrem alterações pré-diabéticas e inflamatórias, ${ }^{16}$ e outros modelos de obesidade em animais apontam para alterações na condução nervosa. ${ }^{9}$ Levando em conta esses fatos, o presente estudo teve como objetivo comparar a nocicepção e, histomorfometricamente, a secção transversa do nervo mediano de ratos Wistar submetidos ao modelo de obesidade MSG, com animais controle.

\section{MÉTODOS}

Todos os protocolos experimentais foram aprovados pelo Comitê de Ética em Uso Animal da Universidade Estadual do Oeste do Paraná (Unioeste), sob número 25/2013-CE. Este estudo caracteriza-se como um ensaio experimental e transversal, no qual foram utilizados ratos Wistar, que ao nascimento receberam uma injeção diária subcutânea de MSG ( $4 \mathrm{~g} / \mathrm{kg}$ de peso corporal/dia - grupo obeso) ou solução salina hiperosmótica $(1,25 \mathrm{~g} / \mathrm{kg}$ de peso corporal/dia - grupo controle), durante os primeiros cinco dias de vida. ${ }^{15}$

Os animais foram mantidos em fotoperíodo claro/ escuro de 12 horas a uma temperatura ambiente de $23 \pm 2^{\circ} \mathrm{C}$, com água e ração ad libitum. Quando completados $94 \pm 4$ dias de idade, os animais foram anestesiados com solução de cloridrato de quetamina $(50 \mathrm{mg} / \mathrm{kg})$ e cloridrato de xilazina $(10 \mathrm{mg} / \mathrm{kg})$, e foi dissecado o nervo mediano direito, logo superior à articulação do cotovelo. Posteriormente, os animais foram eutanasiados por decapitação em guilhotina.

A nocicepção foi avaliada pelo limiar de retirada. ${ }^{17}$ O equipamento utilizado para realizar o teste de sensibilidade dolorosa foi o analgesímetro digital tipo Von Frey da marca Insight ${ }^{\circledR}$ (Ribeirão Preto, São Paulo), o qual consiste de um braço mecânico, com filamento descartável afilado de polipropileno. ${ }^{18}$ Os animais foram contidos manualmente e o filamento de Von Frey aplicado na região palmar da pata anterior direita, com pressão crescente até o momento em que o animal retirava o membro, ou seja, até o limiar de retirada do membro. A primeira avaliação foi realizada cerca de 20 dias antes da eutanásia e a segunda foi realizada no dia anterior ao da eutanásia. Em cada avaliação o teste foi repetido três vezes e utilizado o valor médio das repetições, com unidade em gramas-força (gf).

Para avaliar a obesidade, com o animal anestesiado foi realizado o cálculo do Índice de Lee, por meio da raiz cúbica da massa corporal $(\mathrm{g})$ e o comprimento naso-anal $(\mathrm{cm})$, multiplicado por 1000 . O resultado 
demonstra o estado nutritivo, pois o índice de Lee tem correlação com a massa gorda. ${ }^{19}$ Além desse índice, visando avaliar o modelo de obesidade, após a eutanásia dos animais foi dissecada a gordura das regiões perigonadal e retroperitoneal, que posteriormente foram pesadas em balança analítica.

Foi dissecado o nervo mediano direito, na região do cotovelo, sendo processado com cortes transversais para análise histológica. O processamento histológico foi baseado e adaptado do protocolo de Scipio et al. ${ }^{20}$ Os nervos foram fixados em tetróxido de ósmio 2\% em PBS pH 7,4, emblocados em parafina e cortados transversalmente, com espessura de $5 \mu \mathrm{m}$, em micrótomo CUT 4055 (Olympus, Mainz, Alemanha). As lâminas foram desparafinizadas em xilol, duas vezes de cinco minutos cada, e montadas com Entellan $^{\circledR}$ (Merck, Darmstadt, Alemanha), não havendo necessidade de coloração. Na captura das imagens, os nervos foram subdivididos em quatro quadrantes, sendo fotomicrografados individualmente em microscópio de luz BX60 (Olympus, Tóquio, Japão), com objetiva de 100 vezes. Posteriormente as imagens foram analisadas com auxílio do programa Image-Pro-Plus 6.0, sendo que em todas foi utilizado um zoom de $25 \%$. As variáveis analisadas foram: número de axônios em quatro campos visuais, localizados nas extremidades superior direita, superior esquerda, inferior direita e inferior esquerda; diâmetros dos axônios, das fibras e da bainha de mielina, que foram contabilizadas 50 fibras por nervo, e coeficiente G (razão entre o diâmetro do axônio pelo diâmetro da fibra nervosa).

Os resultados foram expressos e analisados por meio da estatística descritiva e inferencial. Realizou-se o teste de Shapiro-Wilk para avaliar a normalidade dos dados e posteriormente foi utilizado o teste $t$ de Student para amostras independentes, comparando os grupos. Na comparação intragrupo (nocicepção) foi utilizado o teste $\mathrm{t}$ pareado. O nível de significância aceito foi de $5 \%$.

\section{RESULTADOS}

Foram utilizados no estudo 14 ratos Wistar, sendo sete alocados no grupo obeso e sete no grupo controle. Não foram realizadas medidas nos primeiros cinco dias de vida, porém, os animais eram nascidos na mesma época, de ratas normais (sem obesidade), sendo de tamanhos similares. Aos 90 dias de vida, os grupos apresentaram-se bem definidos, com características corporais distintas, indicando que o grupo MSG realmente tornou-se obeso (Tabela 1).
A avaliação da nocicepção mostrou que os animais do grupo MSG apresentavam menor limiar de retirada, tanto na primeira quanto na segunda avaliação, porém na comparação intragrupo não houve diferenças significativas (Tabela 2).

Para os dados histomorfométricos, os resultados não mostraram diferenças significativas entre os dois grupos estudados (Tabela 3).

Tabela 1. Características corporais de 14 ratos Wistar aos 90 dias de vida, sendo que sete foram submetidos ao modelo de obesidade induzida pelo glutamato monossódico (Grupo Obeso) e sete avaliados como controle (Grupo Controle)

\begin{tabular}{|c|c|c|c|}
\hline Características & Grupo Controle & Grupo Obeso & p \\
\hline Peso corporal (g) & $402,4 \pm 28,3$ & $267,4 \pm 59,0$ & $0,0001^{*}$ \\
\hline $\begin{array}{l}\text { Comprimento } \\
\text { naso-anal }(\mathrm{cm})\end{array}$ & $22,5 \pm 1,3$ & $18,2 \pm 2,1$ & $0,0003^{*}$ \\
\hline Índice de Lee & $328,7 \pm 18,6$ & $354,3 \pm 30,4$ & $0,0406^{*}$ \\
\hline $\begin{array}{l}\text { Peso da gordura } \\
\text { perigonadal (g) }\end{array}$ & $1,363 \pm 0,398$ & $2,263 \pm 0,269$ & $0,0002 *$ \\
\hline $\begin{array}{l}\text { Peso da gordura } \\
\text { retroperitoneal (g) }\end{array}$ & $1,713 \pm 0,321$ & $2.031 \pm 0,352$ & 0,0511 \\
\hline
\end{tabular}

Os dados representam a média \pm desvio-padrão.

* Diferença significativa entre os grupos.

Tabela 2. Valores obtidos para o limiar de retirada da pata, ao estímulo pressórico, em 14 ratos Wistar aos 74 e $94 \pm 4$ dias de vida, sendo que sete foram submetidos ao modelo de obesidade induzida pelo glutamato monossódico (Grupo Obeso) e sete avaliados como controle (Grupo Controle)

\begin{tabular}{|lccc|}
\hline \multicolumn{1}{|c}{ Avaliação } & Grupo Controle & Grupo Obeso & p \\
\hline $\begin{array}{l}\text { Avaliação com } \\
74 \text { dias de vida }\end{array}$ & $149,71 \pm 22,62$ & $118,63 \pm 14,25$ & $0,0096^{*}$ \\
\hline $\begin{array}{l}\text { Avaliação com } \\
94 \text { dias de vida }\end{array}$ & $156,96 \pm 31,96$ & $114,29 \pm 11,32$ & $0,0126^{*}$ \\
& $p=0,6889$ & $p=0,4671$ & \\
\hline
\end{tabular}

Os dados representam a média \pm desvio-padrão.

Os valores são apresentados em gramas.

* Diferença significativa.

Tabela 3. Valores para as variáveis da secção transversa dos nervos medianos à análise histológica, em 14 ratos Wistar anestesiados aos $94 \pm 4$ dias de idade, sendo que sete foram submetidos ao modelo de obesidade induzida pelo glutamato monossódico (Grupo Obeso) e sete foram avaliados como controle (Grupo Controle)

\begin{tabular}{|lccc|}
\multicolumn{1}{c}{ Variáveis } & Grupo Controle & Grupo Obeso & p \\
\hline Número de axônios & $77,7 \pm 32,0$ & $73,0 \pm 36,0$ & 0,3999 \\
\hline $\begin{array}{l}\text { Diâmetro de } \\
\text { axônios }(\mu \mathrm{m})\end{array}$ & $2,99 \pm 0,58$ & $2,89 \pm 0,37$ & 0,3511 \\
$\begin{array}{l}\text { Diâmetro da bainha } \\
\text { de mielina }(\mu \mathrm{m})\end{array}$ & $1,15 \pm 0,31$ & $1,59 \pm 0,41$ & 0,4090 \\
$\begin{array}{l}\text { Diâmetro da fibra } \\
\text { nervosa }(\mu \mathrm{m})\end{array}$ & $6,06 \pm 1,11$ & $6,07 \pm 0,94$ & 0,4917 \\
\hline \begin{tabular}{l} 
Coeficiente G \\
\hline
\end{tabular} & $0,49 \pm 0,03$ & $0,48 \pm 0,06$ & 0,2730 \\
\hline
\end{tabular}

Os dados representam a média \pm desvio-padrão. 


\section{DISCUSSÃO}

A obesidade é considerada um importante fator de risco para o desenvolvimento da neuropatia diabética, com forte evidência para a importância de doença microvascular do nervo na patogênese dessa doença. ${ }^{21}$ Davidson et al., ${ }^{9}$ avaliando a progressão da neuropatia periférica em ratos obesos (com dieta rica em gordura) e diabéticos, observaram que ambos foram resistentes à insulina, sendo que desenvolveram déficits nervosos sensitivos, porém, apenas os diabéticos apresentaram déficits motores. Desta forma, buscou-se no presente estudo avaliar se animais pré-diabéticos, obesos, apresentariam diferenças funcionais e morfométricas em nervos medianos, quando comparados com animais eutróficos. Com relação à nocicepção, foi possível observar um menor limiar para os animais obesos, ou seja, os animais do grupo MSG apresentaram-se mais sensíveis ao limiar de retirada, com menores pressões gerando a irritação necessária para que ocorresse a proteção do membro.

A patogênese da neuropatia periférica diabética é complexa e envolve vários mecanismos, como a alteração do metabolismo da cicloxigenase com subsequente produção de prostaglandinas, tendo consequências específicas aos tecidos, como a ativação de reações inflamatórias. ${ }^{6}$ Ainda, o modelo experimental de obesidade por MSG produz alterações teciduais pró-inflamatórias. ${ }^{16}$

Como limitações do presente estudo, pode-se citar a ausência de avaliações de características bioquímicas do processo inflamatório; contudo, Hernandéz-Bautista et al.,22 avaliando animais submetidos ao mesmo modelo de obesidade, observaram que animais jovens apresentavam alterações no metabolismo, com aumento do fator de necrose tumoral- $\alpha$. Além dessa alteração, também encontraram aumento no índice de Lee, triglicérides, colesterol total e níveis de transaminases, com grande alteração na tolerância à glicose e sensibilidade à insulina. Esses achados corroboram os de Roman-Ramos et al., ${ }^{16}$ que citam que tal modelo de obesidade apresenta, além de aumento da resistência à insulina, alterações inflamatórias crônicas observadas em obesos com diabetes tipo 2. Miranda et al. ${ }^{23}$ citam ainda hiperatividade vagal nesse modelo de obesidade, que é característica da obesidade em humanos, ou seja, alteração no equilíbrio do sistema nervoso autônomo, que pode ser originada por lesão de células do núcleo arqueado hipotalâmico e áreas adjacentes, que o MSG produz.

No presente estudo não foram avaliadas características de condução ou função nervosa, porém, devido às diferenças morfométricas corporais observadas entre os animais submetidos ao MSG e os eutróficos, foi possível observar que os primeiros apresentaram menor massa e comprimento nasoanal, porém com maior acúmulo de gordura, o que lhes conferiu diferenças significativas no índice de Lee, indicativo de obesidade. Essas características foram semelhantes às observadas por Souza et al. ${ }^{24} \mathrm{em}$ estudo biométrico de animais MSG. Acreditava-se que podiam ser encontradas diferenças em algumas características do nervo periférico, além da funcional (nocicepção) anteriormente citada. Contudo, não foi observada qualquer alteração, dentro das variáveis analisadas para a morfometria do nervo mediano, ou seja, não houve diferenças significativas para o número de axônios, diâmetros de bainha de mielina, axônio e fibra nervosa, ou quociente $\mathrm{G}$. Tal fato também foi observado por Soares et al..$^{25}$ ao avaliar a densidade de população de neurônios mioentéricos do íleo de ratos obesos MSG comparados com animais controle.

Para a contagem do número de axônios, padronizouse que dentro do campo de observação todas as fibras compreendidas no campo visual seriam contadas, exceto aquelas que tocassem nas bordas superior e esquerda, $\mathrm{o}$ que foi padronizado para todos os animais. O diâmetro da fibra nervosa é considerado como uma importante avaliação para observar regeneração nervosa. ${ }^{26}$ No presente estudo havia a hipótese de presença de fibras com menor diâmetro para o grupo obeso, o que poderia indicar alterações inclusive para o diâmetro da bainha de mielina, podendo refletir a atividade das células de Schwann na fibra nervosa. ${ }^{27}$ Para ambos os grupos, os valores encontrados para o quociente $G$ ficaram próximos a 0,5 , fugindo do indicativo de degeneração axonal (valores baixos) ou degeneração de mielina (valores altos). ${ }^{26,28} \mathrm{Ou}$ seja, não foram observados indícios de degeneração das fibras nervosas, como era esperado para os animais obesos. Salienta-se que não foram analisados aspectos funcionais do nervo mediano, como por exemplo, a eletroneuromiografia, ou aspectos moleculares do mesmo. Sugerem-se futuros estudos incluindo esses aspectos e os níveis glicêmicos dos animais, visto que o modelo de obesidade utilizado induz a quadro de pré-diabetes.

Com base nos parâmetros avaliados, os animais obesos apresentaram menor limiar nociceptivo, porém não houve diferenças morfométricas dos nervos medianos entre animais submetidos ao modelo de obesidade MSG com ratos controle. Assim, o modelo de obesidade utilizado induziu algumas características hipernociceptivas importantes para pesquisas envolvendo modelos de lesão nervosa, sem prévias diferenças morfométricas do nervo periférico. 


\section{REFERÊNCIAS}

1. Luz DMD, Encarnação JN. Vantagens e desvantagens da cirurgia bariátrica para o tratamento da obesidade mórbida. Rev Bras Obesidade, Nutr e Emagrecimento. 2008;2(10):376-83.

2. Molinatti GM, Limone P. Obesity: a challenge for the clinician. Front Diabetes. 1992;11:7-15.

3. Muller HL, Bueb K, Bartels U, Roth C, Harz K, Graf N, et al. Obesity after childhood craniopharyngioma--German multicenter study on pre-operative risk factors and quality of life. Klin Padiatr. 2001;213(4):244-9.

4. Sowers JR, Draznin B. Insulin, cation metabolism and insulin resistance. J Basic Clin Physiol Pharmacol. 1998;9(2-4):223-33.

5. Dobretsov M, Romanovsky D, Stimers JR. Early diabetic neuropathy: Triggers and mechanisms. World J Gastroenterol. 2007;13(2):175-91.

6. Kellogg AP, Cheng HT, Pop-Busui R. Cyclooxygenase-2 pathway as a potential therapeutic target in diabetic peripheral neuropathy. Curr Drug Targets. 2008;9(1):68-76.

7. Won JC, Kim SS, Ko KS, Cha B. Current status of diabetic peripheral neuropathy in Korea: report of a hospital-based study of type 2 diabetic patients in Korea by the diabetic neuropathy study group of the korean diabetes association. Diabetes Metab J. 2014;38(1):25-31.

8. Callaghan BC, Hur J, Feldman EL. Diabetic neuropathy: one disease or two? Curr Opin Neurol. 2012;25(5):536-41.

9. Davidson EP, Coppey LJ, Kardon RH, Yorek MA. Differences and similarities in development of corneal nerve damage and peripheral neuropathy and in diet-induced obesity and type 2 diabetic rats. Invest Ophthalmol Vis Sci. 2014;55(3):1222-30.

10. Obrosova IG. Diabetic painful and insensate neuropathy: pathogenesis and potential treatments. Neurotherapeutics. 2009;6(4):638-47.

11. Olney JW. Brain lesions, obesity, and other disturbances in mice treated with monosodium glutamate. Science (80-). 1969;164(3880): 719-21.

12. Macho L, Ficková M, Jezová D, Zórad S. Late effects of postnatal administration of monosodium glutamate on insulin action in adult rats. Physiol Res. 2000;49(suppl. 1):S79-85.

13. Nagata M, Suzuki W, Iizuka S, Tabuchi M, Maruyama H, Takeda S, et al. Type 2 diabetes mellitus in obese mouse model induced by monosodium glutamate. Exp Anim. 2006;55(2):109-15.

14. Balbo SL, Grassiolli S, Ribeiro RA, Bonfleur ML, Gravena C, Brito MN, et al. Fat storage is partially dependent on vagal activity and insulin secretion of hypothalamic obese rat. Endocrine. 2007;31(2):142-8.

15. Balbo SL, Gravena C, Bonfleur ML, Mathias PC de F. Insulin secretion and acetylcholinesterase activity in monosodium L-glutamateinduced obese mice. Horm Res. 2000;54(4):186-91.

16. Roman-Ramos R, Almanza-Perez JC, Garcia-Macedo R, Blancas-Flores G, Fortis-Barrera A, Jasso EI. Monosodium glutamate neonatal intoxication associated with obesity in adult stage is characterized by chronic inflammation and increased mRNA expression of peroxisome proliferator-activated receptors in mice. Basic Clin Pharmacol Toxicol. 2011;108(6):406-13.

17. Marcioli MAR, Coradini JG, Kunz RI, Ribeiro LDFC, Brancalhão RMC, Bertolini GRF. Nociceptive and histomorphometric evaluation of neural mobilization in experimental injury of the median nerve. ScientificWorldJournal. 2013. http://dx.doi.org/10.1155/2013/476890.

18. Vivancos GG, Verri Jr WA, Cunha TM, Schivo IRS, Parada CA, Cunha FQ, et al. An electronic pressure-meter nociception paw test for rats. Braz J Med Biol Res. 2004;37(3):391-9.

19. Bernardis LL, Patterson BD. Correlation between "Lee index" and carcass fat content in weanling and adult female rats with hypothalamic lesions. J Endocrinol. 1968;40:527-8.

20. Hernández-Bautista RJ, Alarcón-Aguilar FJ, Escobar-Villanueva MDC, Almanza-Pérez JC, Merino-Aguilar H, Fainstein MK, et al. Biochemical alterations during the obese-aging process in female and male monosodium glutamate (MSG)-treated mice. 2014;11473-94.

21. Miranda RA, Agostinho AR, Trevenzoli IH, Barella LF, Franco CCS, Trombini AB, et al. Insulin oversecretion in MSG-obese rats is related to alterations in cholinergic muscarinic receptor subtypes in pancreatic islets. Cell Physiol Biochem. 2014;33(4):1075-86.

22. Souza F de, Marchesini JB, Campos ACL, Malafaia O, Monteiro OG, Ribeiro FB, et al. Efeito da vagotomia troncular em ratos injetados na fase neonatal com glutamato monossódico: estudo biométrico. Acta Cir Bras. 2001;16(1):32-45.

23. Soares A, Schoffen JPF, Gouveia EM de, Natali MRM. Effects of the neonatal treatment with monosodium glutamate on myenteric neurons and the intestine wall in the ileum of rats. J Gastroenterol. 2006;41(7):674-80.

24. Sá JMR De, Mazzer N, Barbieri CH, Barreira AA. The end-to-side peripheral nerve repair Functional and morphometric study using the peroneal nerve of rats. J Neurosci Methods. 2004;136(1):45-53.

25. Lopes-Filho JD, Caldas HC, Santos FCA, Mazzer N, Simões4 GF, Kawasaki-Oyama RS, et al. Microscopic evidences that bone marrow mononuclear cell treatment improves sciatic nerve regeneration after neurorrhaphy. Microsc Res Tech. 2011;74(4):355-63.

26. Mendonça AC, Barbieri CH, Mazzer N. Directly applied low intensity direct electric current enhances peripheral nerve regeneration in rats. J Neurosci Methods. 2003;129(2):183-90. 